\title{
SUSTENTABILIDADE DOS MEIOS DE VIDA E VULNERABILIDADE SOCIOAMBIENTAL: ESTUDOS COMPARTILHADOS ENTRE BRASIL E CUBA
}

\author{
Márcia Aparecida da Silva Pimentel \\ Universidade Federal do Pará (UFPA) \\ mapimentel@ufpa.br \\ Claudio Fabian Szlafsztein \\ Universidade Federal do Pará (UFPA) \\ ioselesz@gmail.com \\ Ofélia Pérez Montero \\ Universidad de Oriente - UO(Cuba) \\ ofeliapm2019@gmail.com \\ Celene Milanés Batista \\ Universidad de la Costa - CUC (Colômbia) \\ celenemilanes@gmail.com
}

\begin{abstract}
RESUMO
Acordos celebrados entre instituições de ensino e fomento à pesquisa entre Brasil e Cuba têm aproximado pesquisadores para debater assuntos relacionados à capacitação de comunidades ao enfrentamento de mudanças ambientais, com destaque àquelas relacionadas aos eventos meteorológicos extremos. Este artigo apresenta uma discussão sobre sustentabilidade dos meios de vida e sugere estratégias para reduzir a vulnerabilidade socioambiental em duas comunidades, uma localizada na Região Norte do Brasil e a outra, na porção Sul-Oriental de Cuba, com base em indicadores qualitativos. Realizou-se revisão bibliográfica e documental, bem como observações nas áreas de estudo. Aplicou-se a Matriz Fortaleza, Oportunidades, Fraquezas e Ameaças (SWOT, em inglês), para obter informações de base local, as quais, após agrupadas nas dimensões do Capital, permitiram a construção de sete indicadores, que revelaram baixa sustentabilidade das comunidades pesquisadas. Com base nos resultados, ressalta-se a importância da ampliação de projetos interinstitucionais e a implementação de políticas públicas eficientes ao fortalecimento dessas comunidades.
\end{abstract}

Palavras-chave: Sustentabilidade dos Meios de Vida. Comunidades locais. Vulnerabilidade.

\section{SUSTAINABILITY OF LIVELIHOODS AND SOCIO-ENVIRONMENTAL VULNERABILITY: SHARED KNOWLEDGE BETWEEN BRAZIL AND CUBA}

\begin{abstract}
Agreements between research institutions both from Brazil and Cuba have been a key-point on the approximation of researchers concerned with matters such as the challenges presented by climate change to communities, mainly those related to extreme weather events. Thus - based on qualitative data - this paper presents a discussion on sustainability livelihoods and suggests some strategies to decrease the vulnerability of two communities, one from the north of Brazil and the other from the south-east part of Cuba. The method applied includes reviewing documents and previous studies and observations of the aforementioned areas. Also, we applied the Matrix Strong Attributes, Opportunities, Weaknesses and Threats (SWOT) to obtain local-based information - which was then separated in groups and indicated low sustainability of both communities regarding the matrices Weaknesses and Threats. Based on the results, we bring forth the relevance of institutional cooperation and implementation of efficient policies to assist and empower both communities.
\end{abstract}

Keywords: Sustainability livelihoods. Local communities. Vulnerability. 


\section{INTRODUÇÃO}

O interesse de pesquisadores em investigar os efeitos das mudanças ambientais sobre comunidades locais, especialmente as relacionadas ao clima, seja por meio de avaliação do papel dessas comunidades na conservação dos sistemas naturais, seja por meio de discussão sobre a vulnerabilidade de grupos sociais que se encontrem em áreas atingidas pelas referidas mudanças, tornou-se cada vez mais amplo em diversos campos da ciência nas últimas décadas. Há estudos que enfocam temas diversos, como o modo de vida das comunidades e sua relação com o uso sustentável dos recursos naturais (HAMSTEAD e QUINN, 2005; PEREIRA e DIEGUES, 2010; LUCA e KUBO, 2015; JONES et al., 2015; LAMSAL et al., 2015; WALI et al., 2017; GALAFASSI et al., 2018), e ainda outros direcionados ao fortalecimento de tais comunidades ao enfrentamento dos efeitos das mudanças ambientais, notadamente as relacionadas aos fenômenos climáticos, com destaque aos estudos de Adger et al (2009); Gero et al. (2011); Jonge et al. (2012); Bele et al. (2013) e Karlsson et al. (2015).

No âmbito dos marcos legais, desde a Conferência das Nações Unidas para o Desenvolvimento e Meio Ambiente (CNUMAD), ocorrida na cidade do Rio de Janeiro em 1992, Brasil e Cuba, acompanhados de outros países, participaram de discussões sobre a construção de um novo paradigma de desenvolvimento, o qual tinha a sustentabilidade como eixo central. A Agenda 21, um dos documentos assinados nessas discussões, é concebida como um instrumento de planejamento que orienta para elaboração de "ações prioritárias, que estabelecem os caminhos preferenciais da construção da sustentabilidade" (BRASIL, 2004).

Os eventos extremos são desvios de um estado meteorológico moderado que, a curto e médio prazo são os mais importantes para serem estudados, devido ao seu potencial de impactos às atividades humanas (MARENGO et al. 2006). Estes ocorrem de muitas formas como enchentes, secas prolongadas, ondas de calor, tufões e tornados. Jacobi e Giatti (2017) acrescentam que as mudanças climáticas aumentam a frequência desses eventos e que, em alguns casos, é impossível para as populações mais vulneráveis se adaptarem aos níveis futuros dessas mudanças. Por isso os autores alertam para a urgência de uma "gestão preventiva e participativa para a governança ambiental dos riscos de desastres naturais que possibilite o desenvolvimento da capacidade adaptativa da sociedade contemporânea" diante desses fenômenos (p. 2), com destaque às mencionadas.

Nesse contexto, esse artigo propõe a realização de uma pesquisa exploratória sobre o tema da sustentabilidade dos meios de vida em comunidades locais, identificando as vulnerabilidades diante das mudanças ambientais, apresentando um exemplo no Brasil e outro em Cuba. Apesar dos diferentes processos de organização dos seus respectivos territórios é possível constatar que os contextos regionais desses países são marcados pela vulnerabilidade social e pela vulnerabilidade urbana, sendo a primeira constatada por Hummell et al. (2016) para a Região Norte do Brasil; e a segunda, por Milanés Batista $(2011,2012)$ para a região costeira de Santiago de Cuba.

$\mathrm{Na}$ escala das comunidades locais, situações de conflitos socioambientais relacionados ao uso de recursos naturais foram apresentadas por Pimentel (2016) e Díaz López (2017), respectivamente localizadas na zona costeira do Estado do Pará e na Província de Santiago de Cuba. A contribuição deste artigo será apresentar indicadores qualitativos para avaliar a sustentabilidade dos meios de vida nessas áreas e sugerir estratégias de redução da vulnerabilidade das comunidades locais. Para isso, contou com o apoio da literatura nacional e internacional e está subdividido em três seções, além desta introdução e das considerações finais. A primeira reúne uma revisão teórica sobre conceitos-chave relacionados ao tema estudado; a segunda descreve os procedimentos metodológicos utilizados nesta pesquisa; e a terceira apresenta os resultados e a discussão sobre os dados investigados.

\section{REVISÃO BIBLIOGRÁFICA}

\section{O quadro referencial sobre o conceito de Sustentabilidade dos Meios de Vida}

O conceito "meios de vida" (livelihoods, em inglês) é utilizado amplamente por estudiosos de vários países desde a Agenda 21. No capítulo 3 deste documento, intitulado "Combate à Pobreza e capacitação dos pobres para a obtenção de meios de sobrevivência sustentáveis" são ressaltados os seguintes temas: importância da criação de políticas voltadas à conservação e à proteção de recursos dos quais dependem esses grupos humanos; e à promoção da subsistência sustentável, por meio da capacitação e do fortalecimento da autonomia de suas comunidades (ONU Agenda 21). Como mencionado, o documento 
representa um marco legal, norteador de pesquisas para a elaboração de políticas públicas e a implementação dessas políticas pelos países signatários.

A sustentabilidade dos meios de vida em comunidades locais tem abrangência multidimensional que, envolve dimensões como o Capital Natural, que se refere à terra, produção, recursos hídricos, florestas, biodiversidade e recursos ecossistêmicos; o Capital Humano, que está relacionado à saúde, nutrição, educação, conhecimentos e habilidades, capacidade de trabalhar e capacidade de se adaptar; o Capital Social, que diz respeito às redes e conexões, às relações de confiança e reciprocidade, aos grupos formais e informais; o Capital Físico, que corresponde à infraestrutura, ferramentas e tecnologia; e o Capital Financeiro, que trata dos estoques e dos créditos (SCOONES, 1998; LUCA E KUBO, 2015).

Com base nessa compreensão, este artigo trata da capacidade de comunidades para obterem acesso aos elementos contidos nos referidos Capitais. Essa ideia é encontrada em pesquisas desenvolvidas por Reed et al. (2006); Fraser et al. (2006); Reed et al. (2013); Lamsal et al. (2015) e Anaya e Espirito-Santo (2018), entre outras. Reed et al. (2006 e 2013) consideram a sustentabilidade dos meios de vida uma ferramenta conceitual necessária à integração entre pesquisador e comunidade, especialmente no processo de identificação de problemas, na busca de estratégias para o bem-estar de seus integrantes. Fraser et al. (2006) propõem uma análise da sustentabilidade dos meios de vida para o empoderamento de comunidades, em estudos de caso realizados no Canadá, em Botswana e no Reino Unido. Lamsal et al. (2015) analisam a importância das áreas úmidas no Nepal para o meio de sustento de comunidades locais, e defendem a importância do conhecimento, das práticas e da participação dessas comunidades para a conservação de recursos naturais. Anaya; Espirito-Santo (2018) destacam o tema referente aos conflitos de uso-recurso entre Áreas Protegidas (AP) e à sustentabilidade dos meios de vida de comunidades quilombolas no norte do Estado de Minas Gerais, no Brasil.

Com base nos resultados dos aludidos estudos, entende-se que o descaso com o tema sustentabilidade dos recursos implica na vulnerabilidade de comunidades, especialmente diante dos efeitos da ação dos fenômenos naturais extremos, da antropização do ambiente e dos conflitos de interesses divergentes dos grupos sociais politicamente hegemônicos.

\section{As comunidades locais, o conhecimento e a conservação de recursos naturais}

O conhecimento das comunidades é construído com a prática, experiência e conhecimento de seus integrantes sobre a natureza, e também por meio de sua cosmovisão. Os saberes sobre os sistemas naturais e sobre as funções desses sistemas auxiliam o fortalecimento de um comportamento resiliente diante de mudanças ambientais (THORNTON; KITKA, 2010; DELGADO-SERRANO et al., 2017), e, assim, podem contribuir em decisões sobre o enfrentamento de novos cenários.

Berkes et al. (1995) consideram que existem aproximações entre o conhecimento científico e o sistema de saberes tradicionais. De acordo com Argueta (2015), tais semelhanças foram reconhecidas a partir do século XX, em diferentes momentos nos quais povos indígenas e não indígenas se organizaram para defender o seu patrimônio cultural e o seu espaço vital. Entretanto, as comunidades que dependem apenas de uma atividade econômica, podem viver em condição de vulnerabilidade diante de situações de conflitos socioeconômicos ou de mudanças climáticas. Este fato foi constatado por Johansson e Isgren (2017) em estudo sobre duas comunidades tradicionais localizadas na Tanzânia em conflito com proprietários de grandes extensões de terras de considerável valor ecológico que seriam destinadas à monocultura de arroz. Da mesma forma, Twomlow et al. (2008), em pesquisa no sul do continente africano, constataram aumento da vulnerabilidade em comunidades locais nos períodos de secas mais frequentes. Também Hanazaki et al. (2013) revelaram situações de insegurança alimentar em famílias isoladas e com pouca diversidade de atividades econômicas no litoral do Brasil.

\section{Sobre a vulnerabilidade e sustentabilidade dos meios de vida}

O uso do termo 'vulnerabilidade' suscita uma explicação. A literatura sobre este tema é vasta e envolve diferentes áreas de estudo. Fraser et al. (2011) comentam, por exemplo, que o tema se incorpora aos 
estudos das ciências humanas (como sociologia, geografia), das ciências naturais (como ecologia, análise de riscos às mudanças climáticas), repercutindo no campo da governança ambiental.

O desenvolvimento de estratégias de adaptação para reduzir a vulnerabilidade ante aos efeitos de mudanças climáticas, e também das consequências de outras alterações ambientais que resultem em impactos sobre a sociedade, é de grande importância. Isso envolve a experiência dos diferentes grupos sociais, por um lado, e o investimento em políticas públicas eficientes, por outro.

Bele et al. (2013) defendem que algumas comunidades têm seus meios de vida vulneráveis, o que as leva à insegurança alimentar. Os autores também relacionam o tema a questões como saúde, desigualdade de gêneros, fragilidade de governança, carência de infraestrutura e educação, falta de acesso apropriado aos recursos e ausência de capacidade para reagir aos eventos extremos.

Wang et al. $(2014,2016)$ contribuem com o tema meios de vida, vulnerabilidade e mudança climática ao analisarem o sistema pastoral do Tibet. Os autores constatam, em suas análises, que, apesar de os pastores terem desenvolvido várias estratégias de adaptação às alterações do clima, o sistema pastoril ainda está ameaçado pela degradação do uso, e recomendam ações como capacitação e fortalecimento comunitário, a criação de cooperativas formais e informais, e promoção de coordenação entre a comunidade e governo local.

\section{Sobre os Indicadores de Sustentabilidade}

Ampla também é a literatura sobre os indicadores de sustentabilidade. Há variados temas relacionados ao conceito e às metodologias aplicadas sobre os quais será feita uma breve apresentação.

De acordo com a Organization for Economic Cooperation and Development (OECD, 1993), um indicador deve ser compreendido como um modelo, cujos valores são utilizados como parâmetros com capacidade de ceder informações sobre a condição de um fenômeno de maneira significativa.

Fraser et al. (2006) e Reed et al (2006) destacam a existência de dois paradigmas para a construção de indicadores de sustentabilidade. Um deles parte de especialistas que elaboram indicadores globais para análise top-down da sustentabilidade e outro nasce de uma aproximação em escala local (basedcomunity), com construções de indicadores numa perspectiva bottom-up. Nesse grupo, identificam-se as Fortalezas, as Fraquezas, Oportunidades e as Ameaças (FOFA). Em inglês essa metodologia é denominada SWOT (Strengths, Weaknesses, Opportunities e Threats), para gerar indicadores qualitativos, os quais serão importantes para implementação de políticas públicas.

A Matriz FOFA (SWOT) oferece inúmeras possibilidades de ser utilizada no diagnóstico para análise dos fatores socioeconômicos, ambientais, jurídicos internos e externos à comunidade e apresentar indicadores qualitativos da sustentabilidade e vulnerabilidade local. O objetivo deste instrumento é oferecer base para a implementação de ações que envolvam os integrantes da comunidade no manejo e gestão do território (DRUMOND, 2002; KUMMER, 2007; MONDAL, 2017).

\section{METODOLOGIA}

\section{Áreas de Estudo}

O Estado do Pará está localizado na Região Norte do Brasil, mais precisamente no Domínio Morfoclimático Amazônico (AB'SABER, 2003). Nesta região, o clima predominante é quente e úmido, com elevada pluviosidade, cujas características favoreceram o desenvolvimento da vegetação da floresta ombrófila densa. Em escalas de detalhe, observam-se diferentes formações vegetais de influência pedológica, litológica, topográfica e fluviomarinha. A biodiversidade de flora e de fauna foi responsável pelos variados meios de vida das comunidades locais da Amazônia. Atualmente, mesmo com a urbanização e a industrialização da região, o número de comunidades tradicionais que dependem diretamente dos recursos naturais dos seus territórios é elevado. 
A comunidade quilombola "Nossa Senhora do Livramento", localizada no município de Igarapé-Açu, no Estado do Pará é formada por setenta famílias (Figura 1) e teve o reconhecimento do seu território quilombola em 2009 (PARÁ, 2009) e certificada em 2013 (BRASIL, 2013).

Figura1 - Igarapé-Açu (PA)- Comunidade Quilombola “Nossa Senhora do Livramento”, 2019.

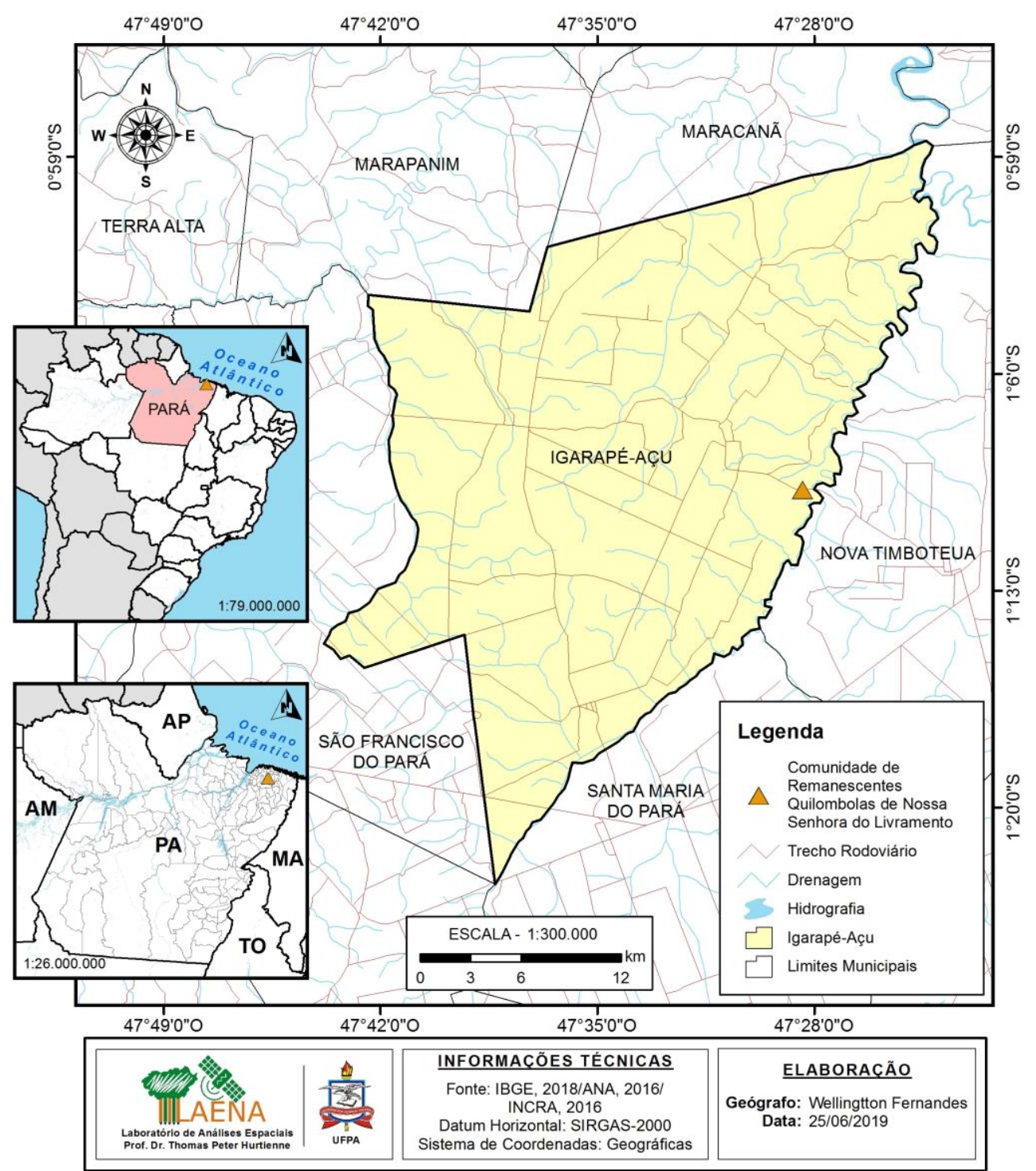

Fonte - Laboratório de Análises Espaciais Prof. Thomas Peter Hurtienne (LAENA), 2019.

A província de Santiago de Cuba (República de Cuba) está localizada na parte sul-oriental da ilha de Cuba. De acordo com Cruz Portorreal (2014), as condições hidroclimáticas nessa região são marcadas por estresse hídrico durante todo o ano e influenciam de forma determinante a configuração das áreas de manguezais. A noroeste da baía desta cidade está a área de manguezal de San Miguel de Parada, a qual, em 2010, foi reconhecida pelo Centro Nacional de Áreas Protegidas/ Sistema Nacional de Áreas Protegidas de Cuba, como Refúgio de Fauna. Atualmente, esta área é administrada pela Empresa Nacional de Proteccíon de Flora y Fauna e abriga a maior diversidade de aves da costa sul-oriental (LAFARGUE HECHAVARRIA, 2010).

Apesar de não haver nenhuma atividade industrial no interior desta unidade, o seu entorno reúne diferentes tipos de indústrias (exemplo. refinarias de petróleo e indústrias alimentícias), cujos dejetos são lançados nos rios que deságuam na baía de Santiago. Essas indústrias, assim como a infraestrutura que servem suas demandas (rodovia e estrada de ferro) constituem-se em fatores de pressão sobre a área de 
conservação e sobre as comunidades localizadas nas proximidades dessas atividades. Três comunidades estão no entorno da Área Protegida: Caimá Chico, Parada e Punta de Sal (Figura 2).

A comunidade de Punta de Sal foi formada, inicialmente, pela população local, mas, ao longo dos anos, e em decorrência do crescimento da cidade de Santiago de Cuba, o fluxo migratório resultou no aumento da população, que conta, atualmente, com cento e vinte quatro moradores (MESTRIL COSME, 2017).

Figura 2 - Santiago de Cuba (Cuba): Área Protegida San Miguel de Parada e a Comunidade Punta de Sal, 2019.

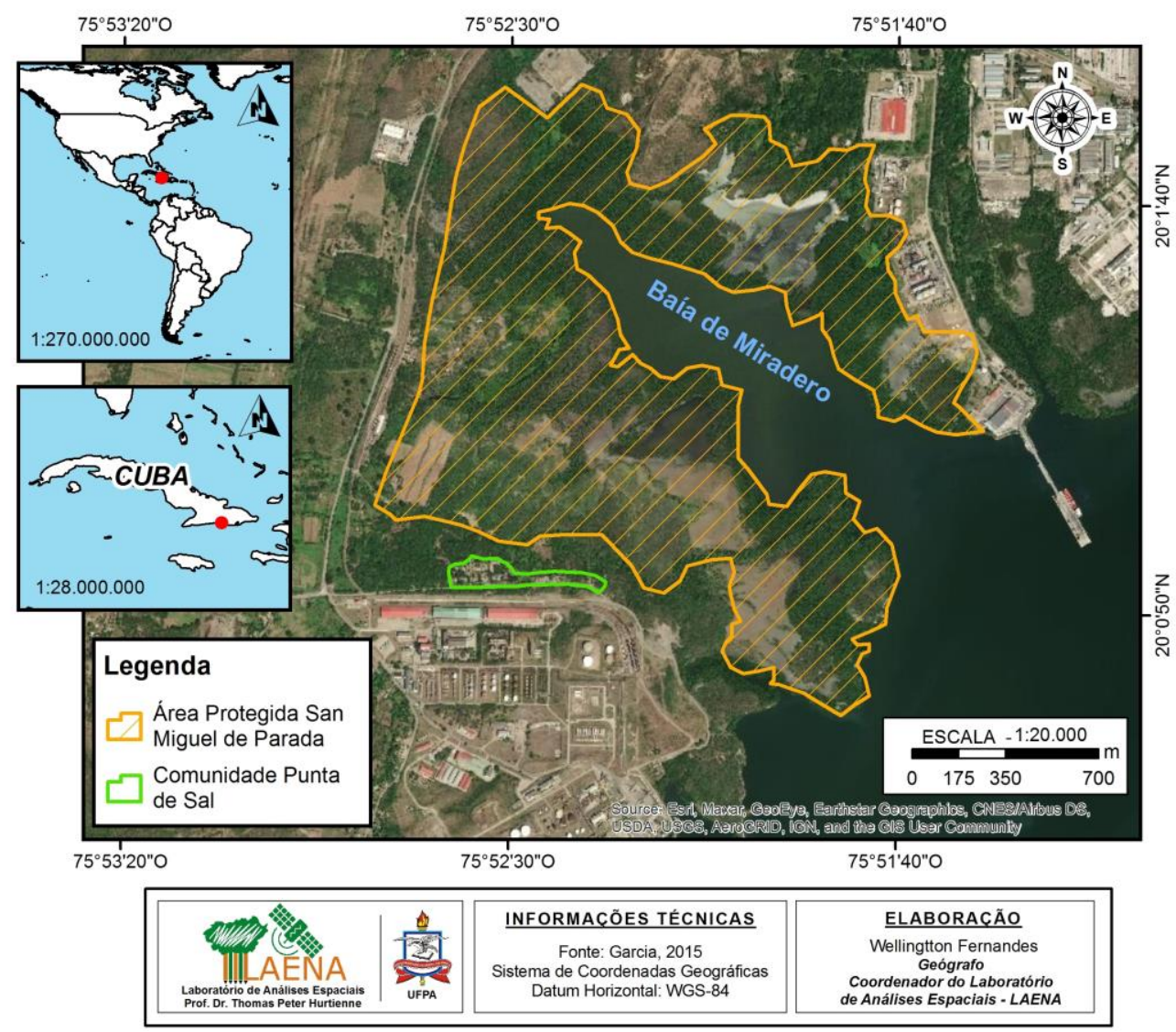

Fonte - Laboratório de Análises Espaciais Prof. Thomas Hurtienne (LAENA/NAEA/UFPA), 2020.

\section{Procedimentos Metodológicos}

No presente artigo utilizou-se o tipo de pesquisa exploratória, a qual se dará com o estudo de dois casos, apresentados anteriormente. Segundo Gil (2006) esta modalidade de pesquisa tem como finalidade proporcionar informações sobre certo assunto, com base em uma visão geral acerca de determinado fato e, em geral, envolve levantamento bibliográfico e documental. A análise das informações obtidas realizada pela aplicação da Matriz FOFA.

Para a construção da Matriz que relaciona as Fortalezas, Oportunidades, Fraquezas e Ameaças foram priorizadas as fontes de dados secundárias constantes em material bibliográfico produzido pelo Grupo de Estudo Paisagem e Planejamento Ambiental (Geppam) da Universidade Federal do Pará e pelo Centro de Estudios Multidiciplinarios da Zona Costera (CEMZOC) da Universidad de Oriente. Nos exemplos apresentados, para o primeiro exemplo, o destaque é dado a Pimentel (2016) e Nascimento e Pimentel (2016). Para o segundo exemplo, recorreu-se a Garcia (2006), Lafargue Hechavarria (2010); Mestril Cosme (2017); Diaz Lopez (2017). A base documental foi fundamentada em Brasil (2007), Pará (2009) e Garcia (2015). A consulta do material, apoiada em um conjunto de questões (Figura 3), norteou a construção de indicadores que possibilitaram a discussão de estratégias de sustentabilidade dos meios de vida das comunidades. 
Figura 3 - Matriz FOFA (SWOT): questões centrais para construção de indicadores.

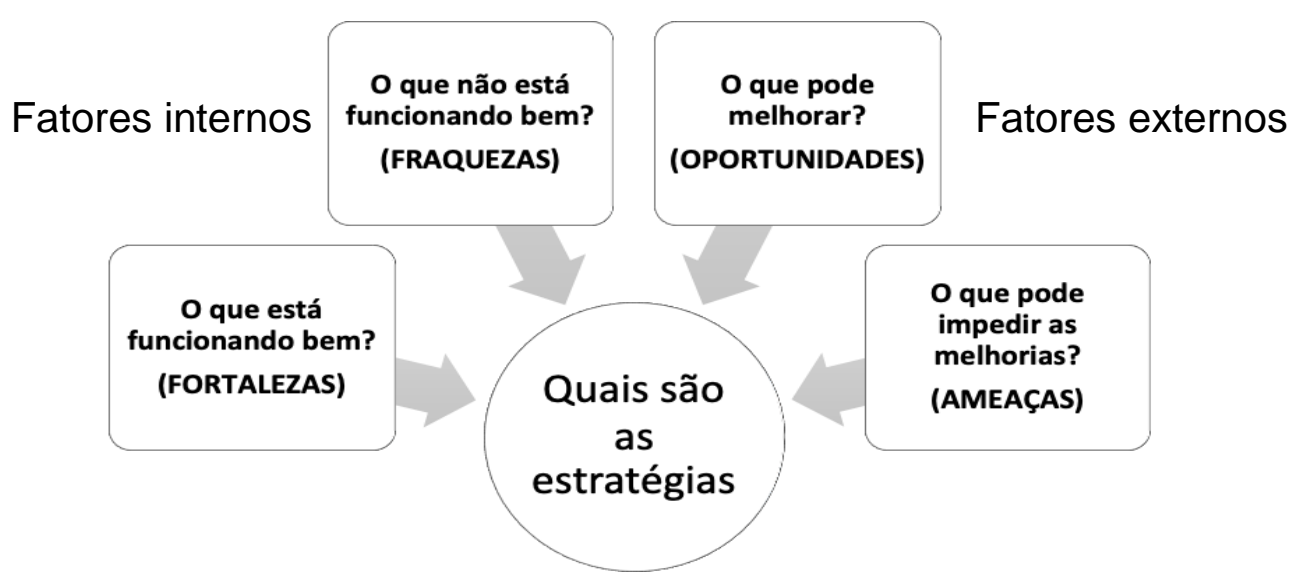

Fonte - Drumond (2002), Kummer (2007) e Mondal (2017).

O esquema com perguntas foi importante para identificar, no material bibliográfico consultado, o diagnóstico socioambiental e econômico para, com base nele, classificar os fatores internos (fortalezas e fraquezas) e externos (oportunidades e ameaças) relacionados às comunidades estudadas. As informações foram sistematizas e agrupadas na Matriz, para proceder a análise qualitativa de acordo com Lamsal et al. (2015).

Além das fontes bibliográficas, destacam-se as técnicas de observação direta, realizadas em pesquisa de campo nos anos de 2016 e 2017 no caso brasileiro, e no ano de 2018 no caso cubano. Durante esses momentos, houve reunião com lideranças comunitárias na comunidade quilombola e em Punta de Sal. Os relatos dos representantes sobre a comunidade corroboraram para atualização das informações adquiridas no material bibliográfico. Contribuíram, ainda, para elaborar os critérios de organização dos indicadores, no contexto de duas das dimensões do Capital: o Capital Natural e o Capital Social e Econômico (SCOONES, 1998).

A análise dos resultados foi feita com base na abordagem qualitativa, um tipo de investigação que, acordo com Marconi e Lakatos (2003), tem como base a descrição, a análise, a interpretação e a atribuição de significados aos fenômenos estudados. Essas etapas foram aplicadas tanto na leitura dos documentos, quanto nas atividades de campo.

\section{RESULTADOS E DISCUSSÃO}

\section{A Comunidade Quilombola “Nossa Senhora do Livramento", Igarapé Açu, Pará}

As comunidades quilombolas são grupos étnicos - predominantemente constituídos pela população negra rural ou urbana - que se autodefinem a partir das relações com a terra, o parentesco, o território, a ancestralidade, as tradições e práticas culturais próprias. A legitimação dos territórios quilombolas é prevista pela Constituição Federal de 1988 (artigos 215 e 216). A identificação, o reconhecimento, delimitação, demarcação e titulação das terras ocupadas por remanescentes de quilombos são regulamentadas pelo Decreto no 4.887 (BRASIL, 2003).

$\mathrm{Na}$ escala regional, a responsabilidade pela determinação de demarcações de suas terras e titulações é do Instituto de Terras do Estado do Pará (ITERPA). Na escala local, as decisões são tomadas pelo Conselho Deliberativo, que reúne os moradores e o presidente do Conselho que, por sua vez, encaminha as reivindicações da comunidade à prefeitura do município. Projetos específicos para esse grupo social têm financiamento da esfera federal. Além disso, seus moradores estão incluídos em políticas públicas do Governo Federal, como os programas de renda mínima conhecidos como Bolsa Família e Minha Casa Minha Vida, que está voltado à habitação popular, ambos para famílias que estão abaixo da linha da pobreza.

A comunidade é formada por famílias que se sustentam por meio de atividades agrárias e de artesanato, cujo trabalho envolve homens, mulheres e crianças De um total de setenta famílias, dez trabalham na lavoura de mandioca, milho e feijão (14\%), três são extrativistas de junco (4\%), um tipo de planta com o qual fazem utensílios para comercialização. As cinquenta e sete famílias restantes (82\%) trabalham com outras atividades em propriedades rurais da região ou na área urbana do município (PIMENTEL, 2016). A construção da Matriz FOFA, para essa comunidade apresentou os resultados expressos na Figura 4. 
Sustentabilidade dos meios de vida e vulnerabilidade socioambiental: estudos compartilhados entre Brasil e Cuba

Figura 4 - Matriz FOFA- Fatores internos (Fortalezas e Fraquezas), fatores externos (Oportunidades e Ameaças) considerados na avaliação de estratégias para sustentabilidade dos meios de vida da comunidade quilombola Nossa Senhora do Livramento.

\begin{tabular}{|c|c|c|}
\hline Fatores de avaliação & \multicolumn{2}{|l|}{ Respostas } \\
\hline $\begin{array}{c}\text { Fortalezas }(\mathrm{F}) \\
\text { O que está funcionando? }\end{array}$ & \multicolumn{2}{|c|}{$\begin{array}{l}\text { F1. Obtenção de título de propriedade da terra quilombola. } \\
\text { F2. Acesso aos programas de construção de casas de alvenaria. } \\
\text { F3. Acesso ao programa de renda mínima - Bolsa Família. } \\
\text { F4. Construção de casa de Farinha de uso coletivo. } \\
\text { F5. Solidariedade entre os moradores. } \\
\text { F6. Criação do Conselho Deliberativo da Associação de Moradores. }\end{array}$} \\
\hline $\begin{array}{c}\text { Fraquezas-(Fq) } \\
\text { O que não está funcionando bem? }\end{array}$ & \multicolumn{2}{|c|}{$\begin{array}{l}\text { Fq1. Falta de apoio técnico para o cultivo da terra. } \\
\text { Fq2. Redução do número de famílias extrativistas e agriculturas. } \\
\text { Fq3.Desmatamento como forma de ampliar as terras agrícolas e aumentar a } \\
\text { produção de mandioca. } \\
\text { Fq4. Família: dependência dos programas sociais de renda mínima. } \\
\text { Fq5. Escola: precária condição do espaço escolar (Ensino Fundamental 1) e } \\
\text { desativação da turma de Educação de Jovens e Adultos. } \\
\text { Fq6. Saúde: ausência de posto de saúde; aumento de casos de gravidez na } \\
\text { adolescência. } \\
\text { Fq7. Disposição inadequada dos resíduos sólidos. }\end{array}$} \\
\hline $\begin{array}{l}\text { Oportunidades }(\mathrm{O}) \\
\text { O que pode melhorar? }\end{array}$ & \multicolumn{2}{|c|}{$\begin{array}{l}\text { O1. Valorização jurídica do conhecimento tradicional. } \\
\text { O2.Editais do Governo Federal para programas de apoio ao desenvolvimento } \\
\text { local. } \\
\text { O3. Projetos de capacitação comunitária e de educação ambiental realizados } \\
\text { pelas Universidades. }\end{array}$} \\
\hline $\begin{array}{c}\text { Ameaças }(\mathrm{A}) \\
\text { O que pode impedir as melhorias? }\end{array}$ & \multicolumn{2}{|c|}{$\begin{array}{l}\text { A1. Pressão de proprietários de terra do entorno da comunidade em } \\
\text { consequência uso da água dos rios. } \\
\text { A2. Demanda por mão de obra barata nos períodos de colheitas das } \\
\text { fazendas produtoras de pimenta. } \\
\text { A3. Exploração de trabalho feminino. } \\
\text { A4. Descontinuidades de políticas públicas locais. } \\
\text { A6. Erosão do solo. } \\
\text { A5. Alterações no ritmo de chuva da região. }\end{array}$} \\
\hline \multicolumn{3}{|c|}{ Estratégias de sustentabilidade dos meios de vida para redução das vulnerabilidades } \\
\hline Oportunidades & $\underline{\mathrm{FO}}$ & $\mathrm{FqO}$ \\
\hline $\begin{array}{l}\text { - O1. Valorização jurídica do } \\
\text { conhecimento tradicional. } \\
\text { - O2. Editais do Governo Federal } \\
\text { para programas de apoio ao } \\
\text { desenvolvimento local. } \\
\text { - O3. Projetos de capacitação } \\
\text { comunitária e educação ambiental } \\
\text { realizados por Universidades. }\end{array}$ & $\begin{array}{l}\text { - Estimular } \\
\text { diversificação da produção } \\
\text { agrícola de pase } \\
\text { sustentável na comunidade } \\
(\mathrm{F} 1, \mathrm{~F} 5, \mathrm{~F} 6, \mathrm{O} 2, \mathrm{O} 3) \text {. } \\
\text { - Criar cooperativa de } \\
\text { produção de produtos } \\
\text { derivados da produção local } \\
(\mathrm{F} 4, \mathrm{~F} 5, \mathrm{~F} 6, \mathrm{O} 1, \mathrm{O} 2) \text {. } \\
\text { - Ter representatividade } \\
\text { nos espaços de discussão } \\
\text { municipal, estadual e } \\
\text { regional (F6, O1, O2, O3). }\end{array}$ & $\begin{array}{l}\text { - Fornecer apoio técnico e capacitação a } \\
\text { agricultores e extrativistas (Fq1, Fq 2, Fq 3, } \\
\text { Fq 4, O2, O3). } \\
\text { - Promover a divulgação de produtos } \\
\text { regionais (Fq4, O1, O2, O3) } \\
\text { - Fortalecer ações de educação ambiental } \\
\text { para reduzir impactos (Fq 3, Fq7, O1, O2, } \\
\text { O3). }\end{array}$ \\
\hline Ameaças & $\underline{\underline{F A}}$ & $\underline{\mathrm{FqA}}$ \\
\hline $\begin{array}{l}\text { - A1. Pressão de proprietários de } \\
\text { terra do entorno da comunidade, } \\
\text { consequência do uso da água dos rios. } \\
\text { - A2. Demanda por mão de obra } \\
\text { barata nos períodos de colheitas de } \\
\text { pimenta nas fazendas. } \\
\text { f3. Exploração do trabalho } \\
\text { feminino. } \\
\text { - A4. Descontinuidades de políticas } \\
\text { públicas locais. } \\
\text { A5. Redução da precipitação no } \\
\text { período chuvoso da região. } \\
\text { - A6. Erosão do solo. }\end{array}$ & 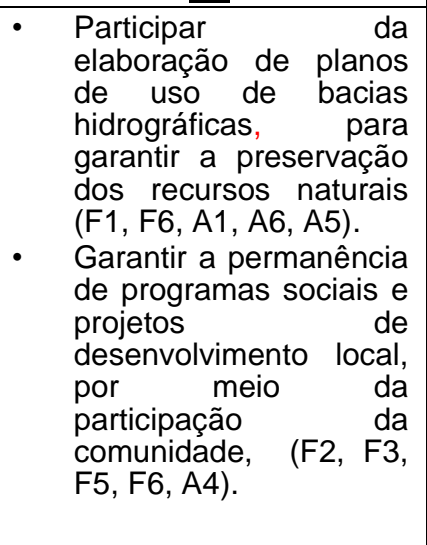 & $\begin{array}{l}\text { - } \quad \text { Elevar a escolarização dos } \\
\text { moradores para redução da } \\
\text { vulnerabilidade social e ambiental } \\
\text { (Fq4, Fq5, Fq6, Fq7, A2, A3, A4). } \\
\text { - } \quad \text { Investir nos setores de saúde e } \\
\text { saneamento básico para reduzir a } \\
\text { exposição a riscos (Fq6, Fq7, A4). } \\
\text { Desenvolver a produção sustentável } \\
\text { de hortaliças e mandioca, por meio } \\
\text { de apoio técnico e de capacitação } \\
\text { comunitária (Fq2, Fq3, Fq4, A2, A3, } \\
\text { A6). } \\
\text { Promover o planejamento ambiental } \\
\text { local e regional com a ajuda de } \\
\text { grupos de interesse (Fq2, Fq3 Fq7, } \\
\text { A1, A4, A6). }\end{array}$ \\
\hline
\end{tabular}

Fonte - Organizada pelos autores, 2019. 


\section{A comunidade de Punta de Sal, Santiago de Cuba}

A comunidade é formada por antigos moradores e por migrantes mais recentes ao lugar. Atualmente alguns deles realizam atividades para a conservação da Área Protegida (LAFARGUE HECHEVARRIA, 2010).

No levantamento realizado por Garcia (2015), a origem da comunidade de Punta de Sal está relacionada ao crescimento e à expansão urbana de Santiago de Cuba, assim como à migração da população da região para a cidade. As famílias utilizam os recursos da área, encontrados no manguezal ou na terra firme, como meio de sustento, seja como alimentação, seja como construção e reconstrução das suas moradias. O aproveitamento ilegal dos recursos florestais na construção de residências e no preparo de alimentos foi constatado, anos atrás, por Garcia (2006), para quem tais atitudes dos moradores são justificadas pela indisponibilidade de outros recursos (materiais para construção, gás de cozinha), o que resulta em impactos sobre a área.

Até 2012, ano da passagem do furacão Sandy em Punta de Sal, existiam quarenta e uma famílias, que viviam na comunidade sem os serviços públicos de água potável, energia elétrica, esgoto sanitário. Mesmo com os danos causados pelo furacão, a comunidade permaneceu no local e, inclusive, teve aquele número aumentado para quarenta e quatro famílias (DIAZ LÓPEZ, 2017). A maior parte dos moradores adultos exerce funções remuneradas na cidade, e apenas uma pequena parte (15\%) presta serviços de manutenção da AP. Na Figura 5 há informações resultantes da Matriz FOFA para a comunidade em discussão.

Figura 5 - Matriz FOFA. Fatores internos (Fortalezas e Fraquezas) e externos (Oportunidades e Ameaças) considerados na avaliação de estratégias para sustentabilidade dos meios de vida da Comunidade de Punta de Sal, 2019.

\begin{tabular}{|c|c|}
\hline Fatores de avaliação & Respostas \\
\hline $\begin{array}{c}\text { Fortalezas }(\mathrm{F}) \\
\text { O que está funcionando? }\end{array}$ & $\begin{array}{l}\text { F1. Estar próximo de uma Área Protegida (AP). } \\
\text { F2. Benefícios adquiridos pelas ações de manejo da } \\
\text { AP, incluindo a educação ambiental. } \\
\text { F3. Envolvimento de parte dos moradores em } \\
\text { atividades de conservação da área. } \\
\text { F4. Escolas de ensino fundamental e serviços de } \\
\text { saúde localizados próximo à comunidade. } \\
\text { F5. Representatividade por meio do Poder Popular } \\
\text { e de organizações políticas, como Comitê de } \\
\text { Defesa da Revolução (CDR) e Federação de } \\
\text { Mulheres Cubanas (FMC). }\end{array}$ \\
\hline $\begin{array}{c}\text { Fraquezas }(\mathrm{Fq}) \\
\text { O que não está funcionando bem? }\end{array}$ & $\begin{array}{l}\text { Fq1. Inexistência de atividades econômicas } \\
\text { desenvolvidas pelos moradores na área ocupada } \\
\text { pela comunidade. } \\
\text { Fq2. Insatisfação da população em relação aos } \\
\text { serviços públicos, como eletricidade e } \\
\text { abastecimento de água. } \\
\text { Fq3. Condições precárias de parte das moradias. } \\
\text { Fq4. Ocorrência de casos de doenças de } \\
\text { veiculação hídrica. } \\
\text { Fq5. Abastecimento de água potável realizado por } \\
\text { caminhões pipa. } \\
\text { Fq6. Uso de madeira, da caça e pesca na Área } \\
\text { Protegida. } \\
\text { Fq7. Insuficiente nível de conhecimento sobre os a } \\
\text { função ecológica da área. }\end{array}$ \\
\hline $\begin{array}{l}\text { Oportunidades }(\mathrm{O}) \\
\text { O que pode melhorar? }\end{array}$ & $\begin{array}{l}\text { O1. Formação de capacidades em Manejo } \\
\text { Integrado de Zonas Costeiras. } \\
\text { O2. Integração entre diferentes instituições de } \\
\text { pesquisa, gestão e fiscalização ambiental. } \\
\text { O3. Política pública de remanejamento dos }\end{array}$ \\
\hline
\end{tabular}


Sustentabilidade dos meios de vida e vulnerabilidade socioambiental: estudos compartilhados entre Brasil e Cuba

\begin{tabular}{|c|c|c|}
\hline & \multicolumn{2}{|c|}{ moradores das áreas contaminadas. } \\
\hline $\begin{array}{c}\text { Ameaças }(\mathrm{A}) \\
\text { O que pode impedir as melhorias? }\end{array}$ & \multicolumn{2}{|c|}{$\begin{array}{l}\text { A1. Crescimento industrial entorno da baía de } \\
\text { Santiago. } \\
\text { A2. Contaminação dos recursos hídricos pelas } \\
\text { empresas existentes. } \\
\text { A3. Aumento dos eventos extremos por efeito das } \\
\text { mudanças climáticas. }\end{array}$} \\
\hline \multicolumn{3}{|c|}{ Estratégias de sustentabilidade dos meios de vida para redução das vulnerabilidades } \\
\hline Oportunidades & FO & $\mathrm{FqO}$ \\
\hline & & - $\quad$ Aumentar o número \\
\hline $\begin{array}{l}\text { - O1. Formação de capacidades em Manejo Integrado } \\
\text { de Zonas Costeiras. } \\
\text { - O2. Integração entre diferentes instituições de } \\
\text { pesquisa, gestão e fiscalização ambiental. } \\
\text { - O3. Remanejamento dos moradores das áreas } \\
\text { contaminadas. }\end{array}$ & $\begin{array}{ll}\text { - } & \text { Ampliar a } \\
\text { participação da } \\
\text { comunidade na } \\
\text { discussão sobre } \\
\text { impactos } \\
\text { socioambientais. } \\
\text { (F1, F2, F3, O1, O2) } \\
\text { - } \quad \text { Fortalecer a } \\
\text { representativida } \\
\text { de na esfera } \\
\text { político- } \\
\text { administrativa } \\
\text { (F5, O3) }\end{array}$ & $\begin{array}{l}\text { de moradores nas } \\
\text { ações de conservação } \\
\text { da Área Protegida } \\
\text { (Fq1, Fq 6, Fq 7, O1, } \\
\text { O2) } \\
\text { Criar condições de } \\
\text { acesso à água potável } \\
\text { para evitar a exposição } \\
\text { dos comunitários aos } \\
\text { os riscos de doenças, } \\
\text { (Fq4, Fq5, O2, O3) }\end{array}$ \\
\hline Ameaças & $\underline{F A}$ & $\underline{F q A}$ \\
\hline $\begin{array}{l}\text { - A1. Crescimento industrial no entorno da baía de } \\
\text { Santiago. } \\
\text { - A2. Contaminação dos recursos hídricos pelas } \\
\text { empresas. } \\
\text { - A3. Aumento dos eventos extremos por efeito } \\
\text { das mudanças climáticas. }\end{array}$ & $\begin{array}{l}\text { Monitorar os } \\
\text { efeitos da } \\
\text { aplicação da } \\
\text { legislação } \\
\text { ambiental sobre } \\
\text { a qualidade dos } \\
\text { recursos } \\
\text { hídricos (F2, F5, } \\
\text { A2). }\end{array}$ & $\begin{array}{c}\text { Melhorar a infraestrutura } \\
\text { física como medida para o } \\
\text { fortalecimento da } \\
\text { comunidade diante dos } \\
\text { efeitos dos fenômenos } \\
\text { atmosféricos extremos } \\
\text { (Fq2, Fq3, Fq7, Fq8, A3). } \\
\text { Equilibrar as ações entre } \\
\text { o crescimento econômico e } \\
\text { o desenvolvimento } \\
\text { sustentável para mitigação } \\
\text { dos impactos } \\
\text { socioambientais (Fq1, Fq2, } \\
\text { Fq3, Fq4, A1, A2). }\end{array}$ \\
\hline
\end{tabular}

Fonte - Organizada pelos autores, 2019.

Como observado, as figuras 4 e 5 apresentam um conjunto de informações importantes para análise das comunidades, pois elencam os seus pontos fortes e as suas debilidades. As estratégias informadas resultaram da análise realizada com base no cruzamento entre os campos (F-Fq-O-A). Nos dois casos pesquisados, o número de itens relacionados às Fraquezas (7) é maior que os de Fortalezas (5), e os de Oportunidades são também, relativamente menores que os de Ameaças. A análise dessa relação demostra, em princípio, um desequilíbrio que antecipa o diagnóstico de vulnerabilidade dos meios de vida. Todavia, quando agrupados, tais campos são envolvidos em dimensões mais amplas, nas quais se incluem os recursos do Capital (natural, físico, humano, social e financeiro) propostos por Scoones (1998) e aplicados por Reed et al. (2006) e Luca; Kubo (2015). A agregação dos temas nessas dimensões favorece a construção de indicadores construídos em dimensão local e referenciados em dimensão global.

Os seguintes indicadores foram observados com base nas informações extraídas das referidas figuras. No campo dos recursos do Capital Natural: 1. Água: acesso e qualidade: nos dois casos apresentados, a água aparece no campo da Ameaça, seja pela contaminação industrial (no caso de Punta de Sal), seja pelo controle ao acesso exercido por outros atores (médios e grandes proprietários rurais do entorno da

$\begin{array}{lllll}\text { Caminhos de Geografia } & \text { Uberlândia-MG } & \text { v. 22, n. } 81 & \text { jun./2021 } & \text { p. 249-264 Página } 258\end{array}$


comunidade quilombola), caracterizando, assim, os conflitos do tipo uso-recurso. No mesmo campo, a água também aparece relacionada diretamente aos efeitos das mudanças climáticas, seja nos episódios de intensos períodos de chuvas ou de secas atípicas. 2. Solo: fertilidade e erosão: no caso da atividade agrícola, a utilização excessiva do solo, associada à falta de acompanhamento técnico, incide sobre a baixa capacidade de produção e, uma vez exposto, associado ao clima chuvoso amazônico, o solo tornase vulnerável à erosão. Por último, nesse indicador, há que se mencionar a poluição resultante da disposição dos resíduos sólidos. 3. Biodiversidade: uso e conservação: ressalta-se, especialmente na Comunidade de Punta de Sal, que a criação da Área Protegida do tipo Refúgio de Fauna é um indicador positivo, seja pela sua função ecológica e estratégica, seja pelo envolvimento da comunidade na formação de capacidades no tema da conservação ambiental. No caso da comunidade quilombola, observa-se que a prática do desmatamento para ampliação das culturas é um impacto negativo e se opõe aos princípios da sustentabilidade das comunidades tradicionais.

No campo do Capital Social e Econômico foram determinados os seguintes indicadores: 4. Educação: acesso, qualidade e continuidade: embora a gratuidade e a qualidade do ensino sejam reconhecidas, especialmente pela inclusão da Educação Ambiental nos programas de ensino no Brasil e em Cuba, no item continuidade dos estudos, observou-se que existem barreiras internas (Fraqueza), como a dificuldade dos jovens para conciliar o trabalho com os estudos noturnos, e barreiras externas (Ameaças), pertinentes a distância entre a comunidade e as instituições públicas que ofertam ensino secundário. 5. Saúde: infraestruturas e exposição ao risco: assim como o indicador anterior, a Saúde, nos dois casos pesquisados, é pública e gratuita. Nota-se, no entanto, que a insatisfação das comunidades está na distância em que se encontram os equipamentos. Em relação à qualidade, ressalta-se, com gravidade, a exposição das comunidades às doenças de veiculação hídrica. No caso de Punta de Sal, isso está demonstrado pela contaminação industrial; no caso da comunidade quilombola, a poluição é proveniente dos vetores de transmissão que se propagam devido à disposição inadequada dos resíduos sólidos. 6 . Proventos e benefícios: este indicador está relacionado à economia das duas comunidades e revelou situações distintas entre ambas. Na comunidade quilombola, por exemplo, os proventos advindos da produção agrícola ou extrativismo são menores porque poucas famílias trabalham a terra. Os motivos pontuados na figura 4 referem-se, sobretudo, ao pouco estímulo e capacitação técnica dos agricultores, o que demonstra incoerência na própria política pública, que prevê o reconhecimento da comunidade rural. Dessa forma, os proventos são adquiridos do trabalho em propriedades externas à comunidade ou de atividades remuneradas realizadas na cidade. Devido a essa situação que gera (e tem gerado historicamente) a pobreza do trabalhador rural, algumas políticas públicas sociais passaram a atender essa população com benefícios de renda mínima. No caso de Punta de Sal, a maior parte dos comunitários é formada por trabalhadores urbanos, e há uma pequena parte que recebe remuneração pelas atividades de manejo da AP. Apesar dos benefícios e da assistência governamental, os moradores não possuem recursos financeiros para, por exemplo, fazer melhorias na estrutura das moradias, conforme apontaram Garcia (2015) e Díaz Lopes (2017). 7. Articulação política: instrumentos e representação: este indicador, tão importante quanto os demais, apresenta-se como positivo para as duas comunidades estudadas, visto que há organização interna dos moradores em associação na comunidade quilombola, e representatividade política do grupo em Punta de Sal, em outros fóruns. Nesse tema, propõe-se, como estratégia, o fortalecimento da participação desses grupos, para aumentar o seu empoderamento e reduzir os conflitos. Nesse aspecto, de acordo com Lucas; Kubo (2015) e Anaya e Espirito-Santo (2018), as instituições públicas e outros órgãos não governamentais exercem um papel fundamental.

$\mathrm{Na}$ análise conjunta dos dois estudos de caso, os sete indicadores apontaram a vulnerabilidade das comunidades onde a questão dos conflitos socioambientais é observável, apesar de seus diferentes contextos político-econômicos. Tanto no primeiro como no segundo caso, os resultados confirmam que a participação das comunidades na conservação ambiental é fundamental, entretanto, é necessário investir em políticas públicas (educação, infraestrutura, trabalho e renda), para reduzir a vulnerabilidade dos sistemas social e ambiental, considerando que esses dois campos não podem ser vistos de forma isolada.

Caminhos de Geografia

Uberlândia-MG

v. 22, n. 81

jun./2021

p. $249-264$

Página 259 


\section{CONSIDERAÇÕES FINAIS}

A sustentabilidade dos meios de vida é compreendida como um conjunto de fatores articulados que está pautado nas diretrizes da Agenda 21 e nos acordos firmados entre vários países, entre os quais, Brasil e Cuba, para melhorar a qualidade de vida de comunidades locais e, assim, reduzir as situações de vulnerabilidades com ações que aumentem a sua capacidade de reconstrução diante de pressões ou ameaças. Dessa forma, entende-se esse tema como pertinente, atual, pois envolve a comunidade científica global, conforme as referências citadas no presente artigo confirmaram.

A metodologia utilizada neste artigo forneceu sete indicadores de base local que permitiram uma discussão sobre questões relacionadas à sustentabilidade e à vulnerabilidade nas comunidades estudadas. O levantamento e a análise do material produzido revelaram um diagnóstico segundo o qual tanto a comunidade quilombola quanto a comunidade de Punta de Sal apresentam frágil ou baixa sustentabilidade dos seus meios de vida. Isso evidencia que ambas apresentam vulnerabilidade diante das mudanças ambientais, não demonstrando preparo para o enfrentamento dos seus efeitos. As estratégias apontam para as ações que chamem a atenção de pesquisadores, de instituições locais e do Estado para o desenvolvimento de projetos em conjunto que fortaleçam a capacidade de gestão das comunidades sobre os recursos necessários à sua reprodução social e econômica, e as levem a entender que, para atingir esse objetivo, a melhor resposta é a implementação de políticas públicas integradas às suas necessidades.

Para finalizar, destaca-se que o presente estudo evidenciou que, nos dois casos analisados, os atores econômicos e políticos são hegemônicos sobre outros fatores que atuam na esfera social e ambiental. Os conflitos são originados pelo projeto de desenvolvimento econômico entendido como crescimento da produção. No caso do Brasil, esse projeto é liderado pelo capital privado e estatal; no caso de Cuba, pelas empresas estatais. Em ambos, este crescimento é ainda compreendido como um dilema a ser resolvido entre a produção e a conservação dos recursos.

\section{AGRADECIMENTOS}

Os autores agradecem à Coordenação de Aperfeiçoamento de Pessoal de Nível Superior (CAPES) pelo financiamento do projeto e aos moradores da Comunidade de Punta de Sal e Comunidade Quilombola de Nossa Senhora do Livramento.

\section{REFERÊNCIAS}

AB'SÁBER, A. Os domínios de natureza no Brasil: potencialidades paisagísticas. São Paulo: Ateliê Editorial. 159 p., 2003.

ADGER W.; DESSAI, S.; GOULDEN, M. HUME, M. NELSON, D.; NAESS, L.; WOLF, J.; WRWFORD, A. Are there social limits to adaptation to climate change? Climatic Change v.93, n.3-4, p. 335-354, 2009. https://doi.org/10.1007/s10584-008-9520-z

ANAYA, F.; ESPIRITO-SANTO, M. Protected areas and territorial exclusion of traditional communities: analyzing the social impacts of environmental compensation strategies in Brazil. Ecology and Society, v. 23, n.1, 2018. https://doi.org/10.5751/ES-09850-230108

ARGUETA, A. Sistemas e saberes ambientais, natureza e construção do bem viver. Desenvolvimento e Meio Ambiente, v. 35, n.3, 2015. https://doi.org/10.5380/dma.v35i0.43544

BELE, M.; TIANI, A.; SOMORIN, O.; SONWA D. Exploring vulnerability and adaptation to climate change of communities in the forest zone of Cameroon. Climatic Change, v.119, n.3-4, p.875-889, 2013. https://doi.org/10.1007/s10584-013-0738-z

BENTLEY BRYMER, A.; HOLBROOK, J.; NIEMEYER, R.; SUAZO, A.; WULFHORST, J.; VIERLING, K.; NEWINGHAM, B.; LINK, T.; RACHLOW. J. A social-ecological impact assessment for public lands

$\begin{array}{lllll}\text { Caminhos de Geografia } \quad \text { Uberlândia-MG } & \text { v. 22, n. } 81 & \text { jun./2021 } & \text { p. 249-264 Página } 260\end{array}$


management: application of a conceptual and methodological framework. Ecology and Society, v. 21, n.3, 2016. https://doi.org/10.5751/ES-08569-210309

BERKES, F.; FOLKES, C.; GADGIL, M. Traditional Ecological Knowledge, Biodiversity, Resilience and Sustainability. In: PERRINGS C. (Ed.) Biodiversity Conservation. Netherlands: Kluwer Academic Publishers, p. 281-299, 1995. https://doi.org/10.1007/978-94-011-0277-3 15

BRASIL. Decreto no 4.887 de 20 de novembro de 2003. Regulamenta o procedimento para identificação, reconhecimento, delimitação, demarcação e titulação das terras ocupadas por remanescentes das comunidades dos quilombos de que trata 0 art. 68 do Ato das Disposições Constitucionais Transitórias. Brasília (DF), 2003.

BRASIL. Agenda 21 brasileira: resultado da consulta nacional / Comissão de Políticas de Desenvolvimento Sustentável e da Agenda 21 Nacional. 2 ed. Brasília (DF): Ministério do Meio Ambiente, 2004. $158 \mathrm{p}$.

BRASIL. Decreto no 6.040 de 7 de fevereiro de 2007. Institui a Política Nacional de Desenvolvimento Sustentável dos Povos e Comunidades Tradicionais. Brasília (DF), 2007.

BRASIL. Portaria no 109 de 30 de julho de 2013. Ministério da Cultura/Fundação Cultural Palmares. Certifica a Autodefinição das Comunidades Remanescentes de Quilombo. Brasília (DF), 2013

CRUZ PORTORREAL.Y. Crecer con los manglares. Editorial Cubasolar, Habana. 2014. 64p.

DELGADO-SERRANO, M.; MISTRY, J.; MATZDORF, B.; LECLERC, G. Community-based management of environmental challenges in Latin America and the Caribbean. Ecology and Society, v. 22, n.1 p.1-9, 2017. https://doi.org/10.5751/ES-08924-220104

DÍAZ LOPÉZ, V. Método para la planificación y evaluación de programas de Manejo Integrado de Zonas Costeras: COPAMIZC. Aplicación en el Refugio de Fauna San Miguel de Parada. Tesis presentada para opción al grado científico de Máster en Manejo Integrado de Zonas Costeras. Centro de Estudios Multidisciplinarios de Zonas Costeras-Universidad de Oriente-Cuba. 2017

DRUMOND, M. Participação Comunitária no Manejo de Unidades de Conservação: Manual de Técnicas e Ferramentas. Belo Horizonte: Instituto Terra Brasilis de Desenvolvimento Socioambiental, 8p, 2002.

FRASER, E.; DOUGILL, A.; MABEE, W.; REED, M.; MCALPINE, P. Bottom-up and top down: Analysis of participatory processes for sustainability indicator identification as a pathway to community empowerment and sustainable environmental management. Journal of Environmental Management, n. 78, 114-127, 2006. https://doi.org/10.1016/j.jenvman.2005.04.009

FRASER, E.; DOUGILL, A.; HUBACEK, K.; QUINN, C.; SENDZIMIR, J.; TERMANSEN, M. Assessing vulnerability to climate change in dryland livelihood systems: conceptual challenges and interdisciplinary solutions. Ecology and Society, v.16, n.3, p. 1-10, 2011. https://doi.org/10.5751/ES-03402-160303

GALAFASSI, D.; DAW, T.M.; THYRESSON, M.; ROSENDO, S. CHAIGNEAU T.; BANDEIRA S.; MUNYI, L.; GABRIELSSON, I.; BROWN, K. Stories in social-ecological knowledge concretion. Ecology and Society, v. 23, n.1, p.1-30, 2018. https://doi.org/10.5751/ES-09932-230123

GARCIA, G. Refugio de Fauna San Miguel de Parada: Plan de Manejo 2016-2020. Santiago de Cuba: Empresa Nacional para la Proteccíon de la Flora y la Fauna e Oficina de Conservación, 2015, 170 p.

GARCIA P. R. Bases para el Manejo Integrado del Bosque de mangles associado al humedal San Miguel de Parada. Tesis em opcíon al Título de Máster em MIZC. Centro de Estudios Multidisciplinarios de Zonas Costeras Universidad de Oriente.Cuba. 2006.

GERO, K.; EHEUX, M.; DOMINEY, D. Integrating community-based disaster risk reduction and climate change adaptation: examples from the Pacific. Natural. Hazards Earth System. Science. n.11, p.101113, 2011. https://doi.org/10.5194/nhess-11-101-2011 


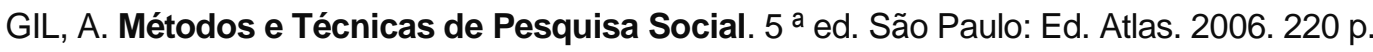

HAMSTEAD, M.; QUINN, M. Sustainable Community Development and Ecological Economics: Theoretical Convergence and Practical Implications. Local Environment. v. 10, n. 2, 141-158, 2005.

https://doi.org/10.1080/1354983052000330743

HANAZAKI, N.; BERKES, F.; SEIXAS, C.; PERONI, N. Livelihood Diversity, Food Security and Resilience among the caiçara of Coastal Brazil. Human. Ecology. v. 41, p. 153-14, 2013.

https://doi.org/10.1007/s10745-012-9553-9

HUMMELL, B.; CUTTER, S.; EMRICH, C. Social Vulnerability to Natural Hazards in Brazil. International Journal Disaster Risk Science. n.7, 111-122, 2016. https://doi.org/10.1007/s13753-016-0090-9

JACOBI P., GIATTI L. L. Eventos extremos, urgências e mudanças climáticas. Ambiente \& Sociedade. v. XX, n. 3, p. 1-6, 2017. https://doi.org/10.1590/1809-4422asoceditorialv2012017

JOHANSSON, E.; ISGREN, E. Local perceptions of land-use change: using participatory art to reveal direct and indirect socio environmental effects of land acquisitions in Kilombero Valley, Tanzania. Ecology and Society, v. 22 n. 1, p. 1-12, 2017. https://doi.org/10.5751/ES-08986-220103

JONES, C.; KIELLAND K.; HINZMAN L.; SCHNEIDER, W. Integrating local knowledge and science: economic consequences of driftwood harvest in a changing climate. Ecology and Society, v. 20, n. 1, p.114, 2015. https://doi.org/10.5751/ES-07235-200125

JONGE, V.; PINTO, R.; TURNERD, R. Integrating ecological, economic and social aspects to generate useful management information under the EU Directives' 'ecosystem approach'. Ocean \& Coastal Management, n. 68, 169-188, 2012. https://doi.org/10.1016/j.ocecoaman.2012.05.017

KARLSSON, M.; VAN OORT, B.; ROMSTAD, B. What we have lost and cannot become: societal outcomes of coastal erosion in southern Belize. Ecology and Society, v. 20, n. 1, p. 1-14, 2015. https://doi.org/10.5751/ES-07050-200104

KUMMER, L. Metodologia participativa no meio rural, uma visão interdisciplinar: conceitos, ferramentas e vivências. Salvador, GTZ, 2007. 155 p.

LAFARGUE HECHAVARRIA, S. Perfeccionamiento de las acciones de manejo de Refugio de Fauna San Miguel de Parada, para su declaración como Zona bajo Régimen de Manejo Integrado Costero. Tesis de Master. Centro de Estudios Multidisciplinarios de Zonas Costeras. Universidad de Oriente, Cuba. 2010. $95 \mathrm{p}$.

LAMSAL, P.; PANT, P; KUMAR, L.; ATREYA, K. Sustainable livelihoods through conservation of wetland resources: a case of economic benefits from Ghodaghodi Lake, western Nepal. Ecology and Society, v. 20, n. 1, p. 1-11, 2015. https://doi.org/10.5751/ES-07172-200110

LUCA, F.; KUBO. R. Meios de vida rurais sustentáveis em um contexto de agricultura de pouso associada à produção de carvão vegetal em comunidades rurais de Biguaçu/SC. Desenvolvimento e Meio

Ambiente, v. 35, p. 367-383, 2015. https://doi.org/10.5380/dma.v35i0.39868

MARCONI, M.; LAKATOS, E. Fundamentos de Metodologia Científica. 5 ed. São Paulo ed. Atlas, 2003. $310 \mathrm{p}$.

MARENGO, J.; MENÉNDEZ, A.; GUETTER, A.; HOGUE, T.; MECHOSO, C. Eventos Hidrometeorológicos Extremos. Caracterización y Evaluación de Métodos de Predicción de Eventos Extremos de Clima y de la Hidrología en la Cuenca del Plata. REGA Revista de Gestão de Água da América Latina, v. 3, n. 2, p. 29-41, 2006.

MILANÉS BATISTA, C. Asentamientos Costeros en la bahía de Santiago de Cuba: estudio de su vulnerabilidad urbana. Revista Arquitectura y Urbanismo, v.32, n.3, p.18-26, 2011.

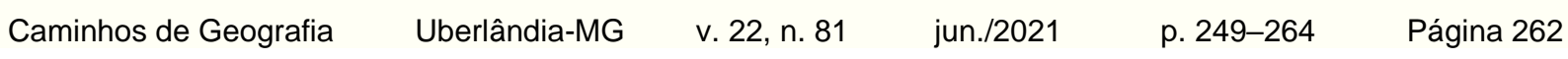


MILANÉS BATISTA, C. Unidades Costeras ambientales para el manejo en Santiago de Cuba: delimitación y prioridades de actuación. Revista Arquitectura y Urbanismo, v.33, n.3, p. 83-97, 2012.

MESTRIL COSME, K. Metodología para incorporar al programa de manejo integrado de zonas costeras el enfoque de valoración económica de bienes y servicios ecosistémicos. Caso Refugio de Fauna San Miguel de Parada. Tesis presentada para opción al grado científico de Máster en Manejo Integrado de Zonas Costeras. Centro de Estudios Multidisciplinarios de Zonas Costeras-Universidad de OrienteCuba, 2017.

MONDAL, S. SWOT Analysis and Strategies to Develop Sustainable Tourism in Bangladesh, UTMS Journal of Economics v. 8, n. 2, 159-167, 2017.

NASCIMENTO, J.; PIMENTEL, M. O uso dos recursos naturais para conservação da biodiversidade no território quilombola de Nossa Senhora do Livramento Igarapé-Açú (PA). Contribuciones a las Ciencias Sociales, v.1, 1-10, 2016.

OECD. Organization for Economic Cooperation and Development. Core set of indicators for environmental performance reviews: a synthesis report by the group on the state of the environment. Paris: OECD, 1993. 38 p.

PARÁ - Instituto de Terra do Pará (ITERPA), 2009. Plano de Utilização da Comunidade Nossa Senhora do Livramento. In: Diário Oficial do Estado do Pará. Publicação DOE 11/11/2009.

PEREIRA, B.; DIEGUES, A. Conhecimento de populações tradicionais como possibilidade de conservação da natureza: uma reflexão sobre a perspectiva da etnoconservação. Desenvolvimento e Meio Ambiente, n. 22, p. 37-50, 2010. https://doi.org/10.5380/dma.v22i0.16054

PIMENTEL, M. Comunidade tradicional e o uso (in)sustentável dos recursos naturais no Nordeste do Estado do Pará Brasil. Contribuciones a las Ciencias Sociales, v.1, p.1-10, 2016.

REED, M.; FRASE, E.; DOUGILL, A. An adaptive learning process for developing and applying sustainability indicators with local communities. Ecological Economic, n.59, 406-418, 2006.

https://doi.org/10.1016/i.ecolecon.2005.11.008

REED, M.; PODESTAL, G.; FAZEY, I.; GEESON, N.; HESSEL, R.; HUBACEK, K.; LETSON, D.; NAINGGOLAN, D.; PRELL, C.; RICKENBACH, M.; RITSEMA, C.; SCHWILCH, G.; STRINGER, L.;THOMAS, A. Combining analytical frameworks to assess livelihood vulnerability to climate change and analyses adaptation options. Ecological Economics, n.94. p.66-67, 2013.

https://doi.org/10.1016/i.ecolecon.2013.07.007

SCOONES, I. Sustainable Rural Livelihoods: A framework for Analysis. Institute of Development Studies n. 72, 1998. 22p.

THORNTON, T.; KITKA, H. The Tlingit Way of Conservation: A Matter of Respect. In Walker Painemilla, K., Rylands, A.; Woofter, A.; Hughes, C. (eds.). Indigenous Peoplesand Conservation: From Rights to Resource Management. Conservation International, Arlington, VA. 2010.

TWOMLOW, S.; MUGABE, F.; MWALE, M.; DELVE, R.; NANJA, D.; CARBERRY, P., HOWDEN, M. Building adaptive capacity to cope with increasing vulnerability due to climatic change in Africa- A new approach. Physics and Chemistry of the Earth. n. 33. p.780-787, 2008.

https://doi.org/10.1016/j.pce.2008.06.048

WALI, A; ALVIRA, D; TALLMAN, P.; RAVIKUMAR, A; MACEDO, M. A new approach to conservation: using community empowerment for sustainable well-being. Ecology and Society, v. 22, n.4, p. 1-3, 2017. https://doi.org/10.5751/ES-09598-220406 
WANG, Y.; WANG J.; LI, S.; QIN, D. Vulnerability of the Tibetan Pastoral Systems to Climate and Global Change. Ecology and Society, v. 19, n. 4, p 1-15, 2014. https://doi.org/10.5751/ES-06803-190408

WANG, J.; Y. WANG, S. LI; D. QIN. Climate adaptation, institutional change, and sustainable livelihoods of herder communities in northern Tibet. Ecology and Society, v. 21, n 1, p. 1-13, 2016.

https://doi.org/10.5751/ES-08170-210105

Recebido em: 21/06/2020

Aceito para publicação em: 28/09/2020 